\title{
A NOVEL HIGH-DENSITY CAPACITOR DESIGN AND ITS FABRICATION TECHNIQUE BASED ON SELECTIVE ETCHING \\ Victor Farm-Guoo Tseng ${ }^{1 *}$, Khai $\mathrm{Ngo}^{2}$, and Huikai Xie ${ }^{l}$ \\ ${ }^{1}$ University of Florida, Gainesville, Florida, USA \\ ${ }^{2}$ Virginia Polytechnic Institute and State University, Blacksburg, Virginia, USA
}

\begin{abstract}
The design, fabrication, and characterization of a novel high-density metal-insulator-metal (MIM) multilayer capacitor is reported. In order to interconnect the multiple electrode layers without using numerous photolithography steps, a unique process was developed based on depositing MIM layers onto a substrate with two pillars, fine polishing to expose the multilayer cross sections, and then selectively etching the metals on each pillar to form the capacitor electrodes. For demonstration purpose, only capacitors with two dielectric layers were fabricated, with measurement results verified by a theoretical model. With a dielectric thickness of $200 \mathrm{~nm}$, a capacitance density of $0.54 \mathrm{fF} / \mu \mathrm{m}^{2}$ was achieved, which can be easily increased by decreasing dielectric thickness and increasing the number of MIM layers. The capacitor also exhibits low equivalent series resistance (ESR) of $300-700 \mathrm{mohms}$, and can operate up to $63 \mathrm{MHz}$.
\end{abstract}

\section{INTRODUCTION}

There has been a great effort in the recent decade to integrate discrete passive devices into the chip level. As mentioned in the recent ITRS report [1], this trend has mainly been influenced by radio frequency (RF), mixed signal, and system-on-a-chip (SoC) applications, in which on-chip precisely controlled high quality passives are needed for high frequency operation. Similarly, in the field of power electronics, minimization of the bulky power passives, possibly fabricated into a separate passives integrated substrate for further flip-chip bonding or 3D chip stacking [2], is necessary. In the overall system, capacitors and inductors are usually the most space consuming, but are rather desired due to their low power consumption. As a result, it is imperative to develop better chip level integration methods for passive components.

In particular, capacitors are the most widely used passive component. Typical applications are microprocessor decoupling capacitors, analog/filter capacitors, RF bypass capacitors, DRAM storage cell capacitors, and power electronic flying capacitors. Currently, several technologies have been developed to fabricate integrated high-density capacitors, including trench capacitors [3], stacked capacitors [4], hemispherical silicon electrode capacitors [5], and multilayer capacitors [6]. While using multilayer stacks seems straightforward, the interconnection between numerous electrode plates requires numerous photolithography steps (Fig. 1(a)), which is impractical and over expensive. Also, the trench capacitor and hemispherical silicon electrode capacitor methods usually make use of the highly doped silicon substrate as one of the capacitor plates, which is undesirable because of the increased substrate coupling noise and loss, along with higher ESR. Thus, there is an increasing demand for MIM capacitors, and for them to be integrated into the back-end-of-line (BEOL) layers of typical IC processes, farther away from the substrate [1].

In this work, a new method is proposed to resolve the interconnection dilemma of multilayer MIM capacitors. This new method will allow the parallel stacking of several tens of MIM capacitors. Additionally, all the previously mentioned high-density

Travel support has been generously provided by the Transducers Research Foundation (TRF) capacitor fabrication techniques can also be incorporated into this technology.

\section{DESIGN CONCEPT}

Illustrated in Fig. 1(b), the underlining concept of our integrated capacitor design and fabrication is based on the idea of subsequently depositing multiple thin film MIM layers onto pillar structures, then polishing down the pillars, and exposing the multilayer cross sections in a single planar horizontal surface, which virtually "bends" the cross sections upwards. If the alternating capacitor electrode metal plate materials are chosen to have chemical properties that allow them to be selectively etched, two selective etch steps can be carried out, one on either one of the pillars, receding either metal 1 or metal 2 down into the trenches on each pillar, as shown in Fig. 1(c). Finally, by performing dielectric redeposition and etch back, followed by electrode metallization, several tens of electrode plates can be interdigitally connected, forming a single capacitor with only four photolithography steps.

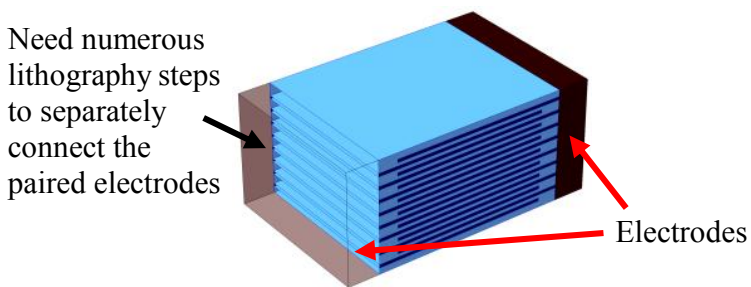

(a)

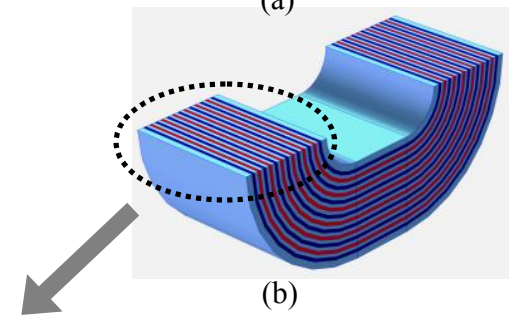

(b)

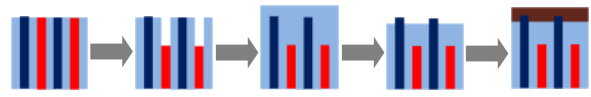

(c)

Dielectric $\quad$ Metal $1 \quad$ Metal 2 Electrode metal

Figure 1. Concept of multilayer capacitor design: (a) typical multilayer capacitors, (b) "bending" the layers upward to access the cross sections while using alternating plate materials, and (c) using selective metal etch, dielectric redeposition and etch-back, and electrode metallization to form the interdigital connections

\section{FABRICATION PROCESS AND RESULTS}

The physical implementation of the fabrication process flow is shown in Fig. 2. First, the pillar structures with height of around $20 \mu \mathrm{m}$ are formed by etching into the silicon substrate. A hydrofluoric, nitric, and acetic acid mixture (HNA) isotropic etch [7] was used to produce smooth pillar sidewalls, which can prevent 
shortage or field concentration at sharp edges. The HNA composition was $\mathrm{HF}(49 \%): \mathrm{HNO}_{3}(70 \%): \mathrm{CH}_{3} \mathrm{COOH}(99.5 \%)=1: 3: 8$, and $\mathrm{Si}_{3} \mathrm{~N}_{4}$ was used as the etch mask. Notice that the pillars can also be made out of the thick low-k dielectrics in typical BEOL layers, but pillars of silicon were chosen for process convenience. Next, a multi-target sputtering system is used to alternatively deposit dielectric, metal 1, dielectric, and metal 2 layers. The dielectric may be $\mathrm{SiO}_{2}, \mathrm{Si}_{3} \mathrm{~N}_{4}$ or other high-k dielectrics. The first and last dielectric layers are deposited by chemical vapor deposition (CVD) to thicknesses around $1 \mu \mathrm{m}$ as isolation/protection layers. The two metals must be chosen to be selectively etched. $\mathrm{Au}$ (with $\mathrm{Ti}$ adhesion) and $\mathrm{Cr}$ were chosen in this work, both sputtered to thicknesses of $500 \mathrm{~nm}$. Then, the pillars are polished down to the first dielectric layer. A combination of polyurethane polishing cloth, $0.3 \mu \mathrm{m}$ alumina lapping film, and $0.05 \mu \mathrm{m}$ colloidal silica/alumina suspension slurry was used to achieve smooth and uniform polishing across the wafer. As shown in Fig. 2(c), photoresist is also spin coated to protect unwanted areas from being attacked during the polishing. Next, the two selective metal etch steps are performed. Gold Etch TFA and Chromium Etch 1020 from Transcene $(\mathcal{C}$ were used respectively for $\mathrm{Au}$ and $\mathrm{Cr}$, and both etchants were shown to be greatly selective to all the other materials. A short Ti etch with a HF based solution is also needed after the Au etch to avoid shortage due to the remaining adhesion layers. The selective etch steps were precisely controlled to recede the metal layers down into the trenches of about $7 \mu \mathrm{m}$. $\mathrm{SiO}_{2}$ is then redeposited by CVD to refill trenches, followed by a short RIE etch back with $\mathrm{CHF}_{3}$ based plasma. Finally, a $300 \mathrm{~nm} \mathrm{Cu}$ seed layer (with $\mathrm{Ti}$ adhesion) is sputtered, molded by photolithography, electroplated to around $10 \mu \mathrm{m}$, and etched back to form the two electrodes. This thick $\mathrm{Cu}$ electrode is needed to achieve lower ESR. For demonstration purpose, only capacitors with two dielectric layers were fabricated: one $200 \mathrm{~nm}$ thick $\mathrm{Si}_{3} \mathrm{~N}_{4}$ dielectric layer, and one $200 \mathrm{~nm}$ thick $\mathrm{SiO}_{2}$ dielectric layer.

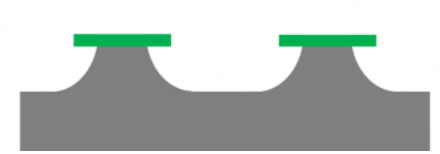

(a)

(b)

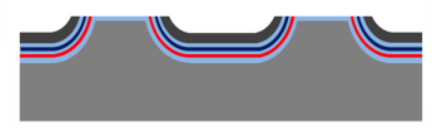

(c)

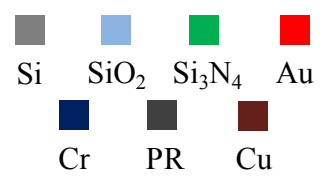

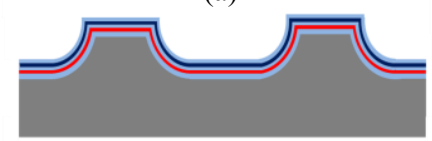

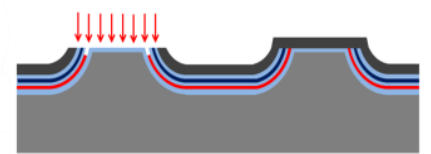

(d)

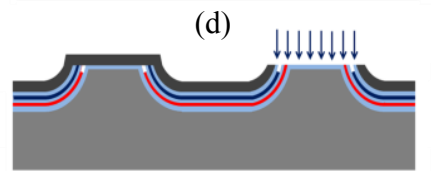

(e)

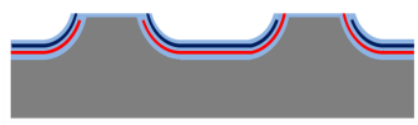

(f)

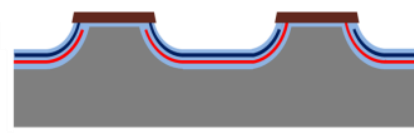

(g)
Figure 2. Complete process flow: (a) HNA etch to form the pillars, (b) multilayer deposition, (c) pillar polishing, (d) selective Au etch, (e) selective $\mathrm{Cr}$ etch, (f) dielectric redeposition and etch back, and (g) sputter and electroplating metallization for $\mathrm{Cu}$ pads

Capacitors were fabricated with areas of $300 \mu \mathrm{m} \times 800 \mu \mathrm{m}$, $800 \mu \mathrm{m} \times 800 \mu \mathrm{m}, 800 \mu \mathrm{m} \times 1800 \mu \mathrm{m}$, and $1800 \mu \mathrm{m} \times 1800 \mu \mathrm{m}$. Fig. 3 shows the two smaller sized completed capacitors. Slight scratches on the first dielectric layer were seen after the polishing process.

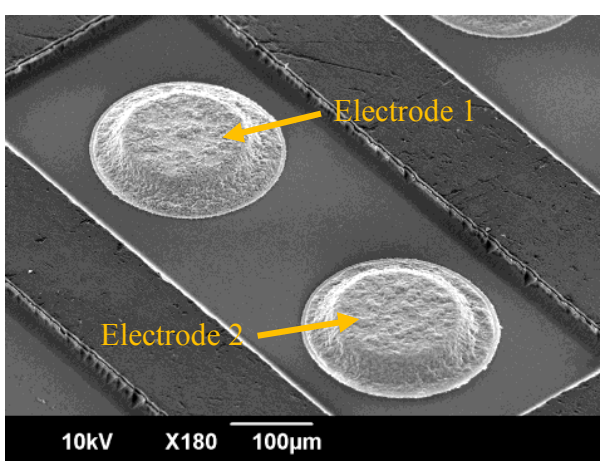

(a)

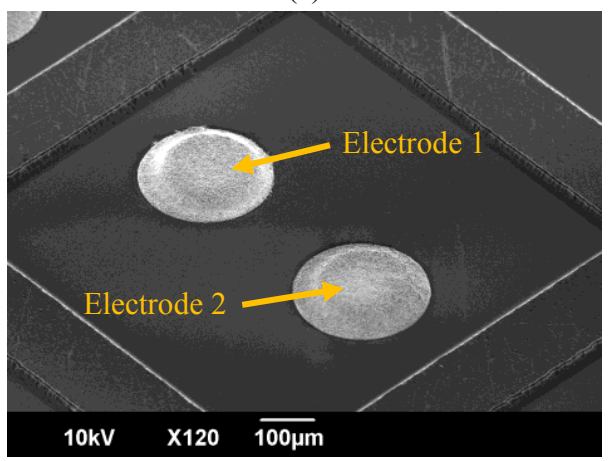

(b)

Figure 3. Completed capacitors with areas of (a) $300 \mu \mathrm{m} \times 800 \mu \mathrm{m}$ and (b) $800 \mu \mathrm{m} \times 800 \mu \mathrm{m}$

Fig. 4 shows an HNA-etched pillar before and after polishing. Clearly, some etching anisotropy exists, and the HNA composition needs to be further tuned to produce a smoother etched surface for the pillars etched out of the high resistivity silicon substrate.

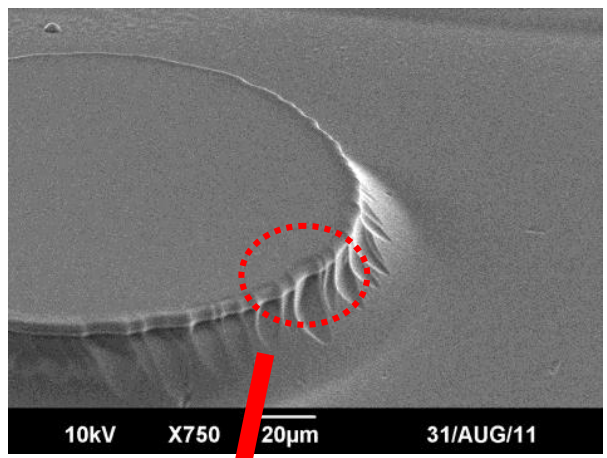

(a)

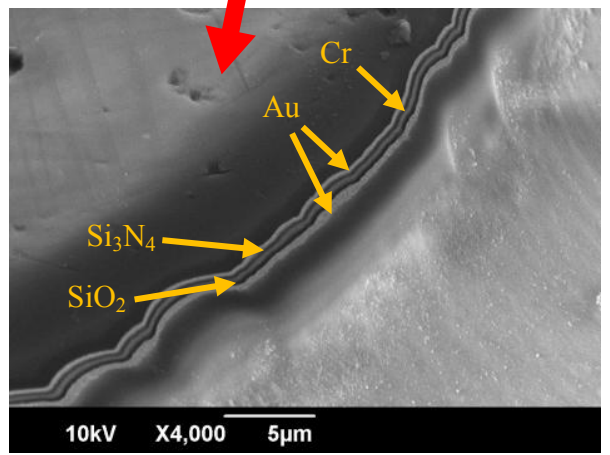

(b)

Figure 4. (a) HNA etched pillar, (b) close-up view showing cross section of a two dielectric layer capacitor after polishing 
Fig. 5(a) shows the selective $\mathrm{Cr}$ etch details. The inner $\mathrm{Cr}$ layer recedes into the cross section, leaving out a trench, while other materials remain intact. Fig. 5(b) shows the results after dielectric redeposition and etch back. The trenches are shown to be refilled and slightly etched. The selective Au etch shows similar results.

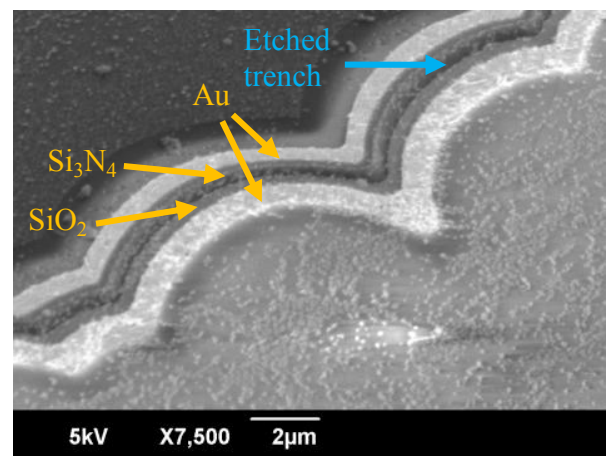

(a)

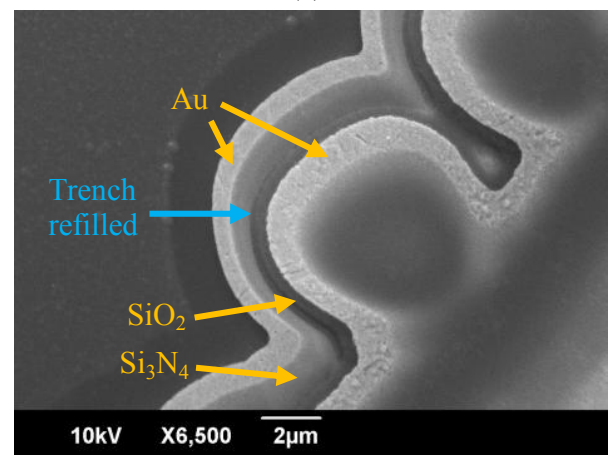

(b)

Figure 5. Close-up view of process details after (a) selective $\mathrm{Cr}$ etch, (b) $\mathrm{SiO}_{2}$ redeposition and etch back

\section{CAPACITOR MODEL}

Shown in Fig. 6 is the typical lumped circuit model for realistic capacitors. $C_{o}$ is the capacitor capacitance, $R_{p}$ is the parallel resistance due to leakage, and $\mathrm{L}_{\mathrm{s}}$ is the equivalent series inductance (ESL) due to the electrodes and plates. $\mathrm{C}_{\mathrm{a}}$ and $\mathrm{R}_{\mathrm{a}}$ are used to fit the response of dielectric absorption, a known result due to polarization hysteresis. $C_{a}$ is usually small compared to $C_{0}$, while $R_{a}$ is large, and both can be neglected in this work. The equivalent series resistance (ESR) includes two components: $\mathrm{R}_{\text {plate }}$, the electrode and plate resistance, which is constant at low frequencies but rises at high frequencies due to skin effect; $R_{d}$, the dielectric loss resistance, which is frequency dependent and has no DC value.

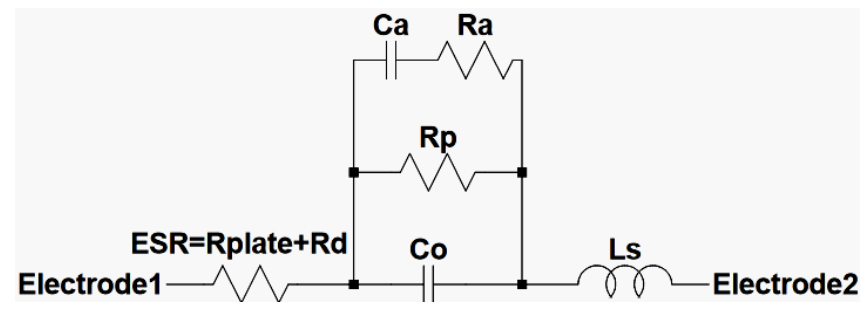

Figure 6. Typical lumped circuit model for realistic capacitor

Assuming a sinusoidal input signal, and defining the complex permittivity as $\epsilon=\epsilon^{\prime}-\mathrm{j} \epsilon^{\prime \prime}$, the loss tangent of the dielectric material then becomes

$$
\tan \delta=\frac{\omega \epsilon^{\prime \prime}+\sigma}{\omega \epsilon^{\prime}} \cong \frac{\epsilon^{\prime \prime}}{\epsilon^{\prime}} \quad \text { (at frequencies above } 500 \mathrm{~Hz} \text { ) }
$$

in which $\sigma$ is the conductivity of the dielectric material. Hence for typical dielectrics, $R_{d}$ can be simplified as

$$
\mathrm{R}_{\mathrm{d}}=\frac{\tan \delta}{\omega \mathrm{C}_{\mathrm{o}}}
$$

Notice that $R_{d}$ takes into account the conductivity of the dielectric, therefore incorporating $R_{p}$ into $R_{d}$. The impedance and dissipation factor (DF) are then expressed as

$$
\begin{gathered}
Z=R_{\text {plate }}+R_{d}+\frac{1}{j \omega C_{o}}+j \omega L_{s} \\
D F=\omega C_{o} \times\left(R_{\text {plate }}+R_{d}\right)
\end{gathered}
$$

\section{MEASUREMENT RESULTS}

Measurements were conducted via a HP 4294A impedance analyzer and an Agilent 4155C parameter analyzer. Measurements of the $300 \times 800 \mu \mathrm{m}$ size capacitors are not shown below due to an existing $\mathrm{k} \Omega$ resistive path through the silicon substrate, mainly caused by over-polishing induced damage to the first dielectric layer. An improved polishing process is needed. The measured capacitances, shown in Fig. 7, were 1 to 1.1 times their expected values. This is due to the increased surface area caused by the HNA etch roughness. The largest capacitance density that is achieved is $0.5 \mathrm{fF} / \mu \mathrm{m}^{2}$, which is comparable to the results in [6]. Shown in Fig. 8, ESR decreases roughly inversely proportional to frequency at first, during which it is dominated by $\mathrm{R}_{\mathrm{d}}$, until it reaches the minimum resistance of $R_{\text {plate }}$. $R_{\text {plate }}$ was found to be around 300 to $700 \mathrm{mohm}$, which is near expectation using equations from [8]. There seems to be an optimal design for capacitor dimensions to minimize $R_{\text {plate}}$, which is still under investigation. More insight can be seen from the DF measurements (Fig. 9). Before around $500 \mathrm{~Hz}$, the higher loss is dominated by the leakage conductivity of the dielectric material. At mid-frequencies, the loss reaches its minimum, dominated by dielectric loss, with $\mathrm{DF} \cong \tan \delta \cong \epsilon^{\prime \prime} / \epsilon^{\prime} \cong 0.003$. At frequencies above several $\mathrm{MHz}$, the loss rises again, dominated by $\mathrm{R}_{\text {plate. }}$. Using (1), the conductivity $\sigma$ of the dielectric material was found to be around $2 \times 10^{-11}(\Omega \times \mathrm{cm})^{-1}$, which corresponds to the $|\mathrm{I}|-\mathrm{V}$ measurements, and is rather large compared to normal dielectric conductivities. In order to improve the dielectric quality, an annealing step can be added. From the impedance magnitude $|\mathrm{Z}|$ measurements (Fig. 10), the self resonances occur at $36.1 \mathrm{MHz}$, $48.6 \mathrm{MHz}$, and $63 \mathrm{MHz}$ respectively, and from (3), $\mathrm{L}_{\mathrm{s}}$ can be found to be $9.6 \mathrm{nH}$. With the extracted model parameters mentioned above, the theoretical response of the lumped circuit model was plotted, and shows close coherence with the measurement results.

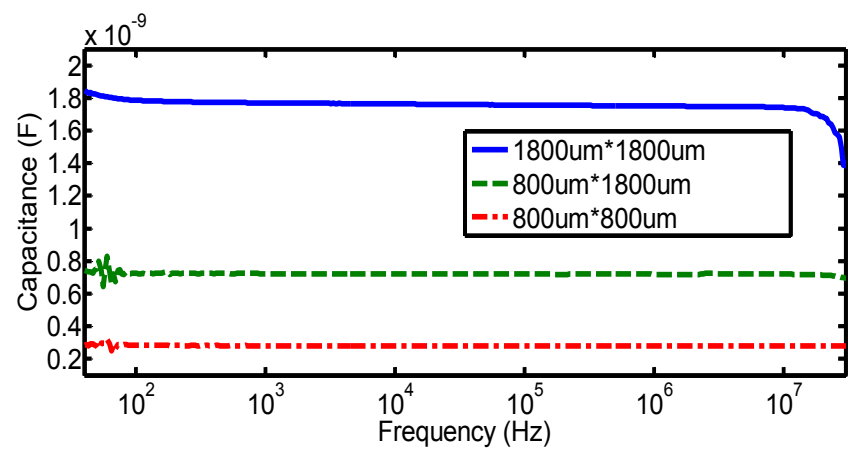

Figure 7. Capacitance measurement results

Fig. 11(a) shows the C-V measurements for the $1800 \mu \mathrm{m} x$ $1800 \mu \mathrm{m}$ size capacitors at different frequencies, showing the capacitance to be constant per bias voltage. Fig. 11(b) shows the $|\mathrm{I}|-\mathrm{V}$ measurements. As mentioned above, a higher than expected 
leakage current due to the higher dielectric conductivity was observed. Occasionally, a premature breakdown voltage at around 2.7 to $3 \mathrm{~V}$ was also observed. Reasons for this might include the inferior dielectric quality, surface roughness induced field concentration, or possible air voids caused by the incompletely filled trenches during the dielectric redeposition step. Further improvements of the dielectric redeposition by using atomic layer deposition (ALD) may help.

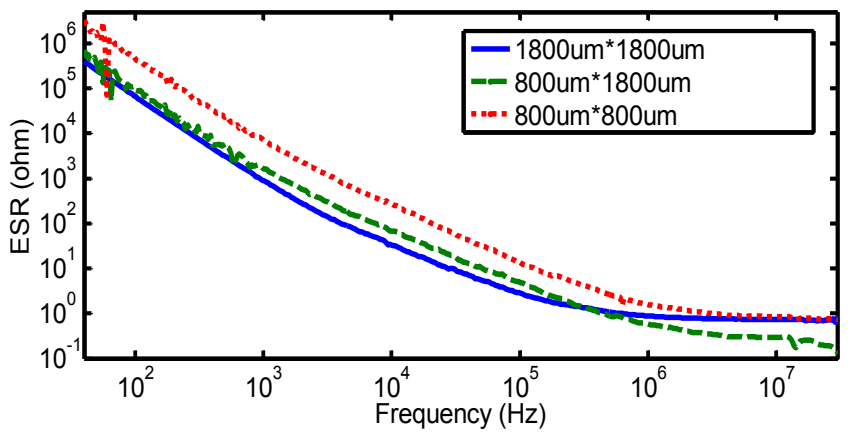

Figure 8. ESR measurement results

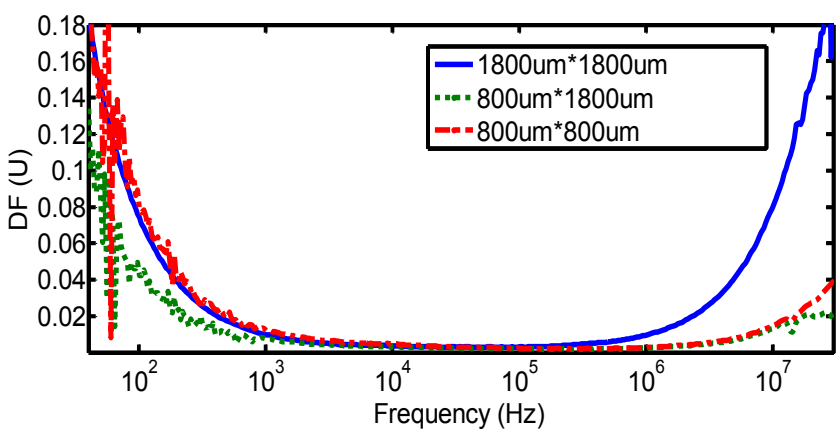

Figure 9. DF measurement results

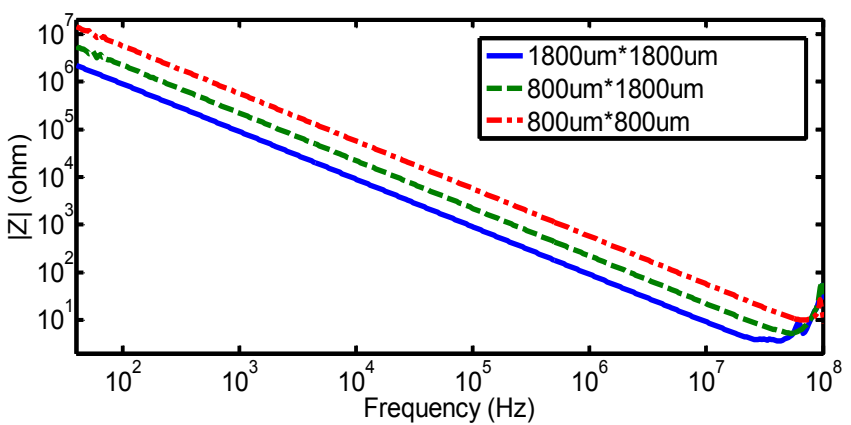

Figure 10. Impedance magnitude measurement results

\section{CONCLUSIONS}

A new method to fabricate high-density multilayer MIM capacitors has been successfully demonstrated, with capacitor performance verified by theory. Further process and design improvements should allow capacitance densities above $12 \mathrm{fF} / \mu \mathrm{m}^{2}$ with lower losses. Since the fabrication is IC-compatible, this innovation should bring benefit to the fields of RF, mixed-signal, power electronics, etc., to achieve the ultimate goal of SoC design.

\section{ACKNOWLEDGEMENTS}

This project is supported by ARPA-E under award \#DE-AR0000105. Device fabrication and characterization was done in the Nanoscale Research Facility of the University of Florida.

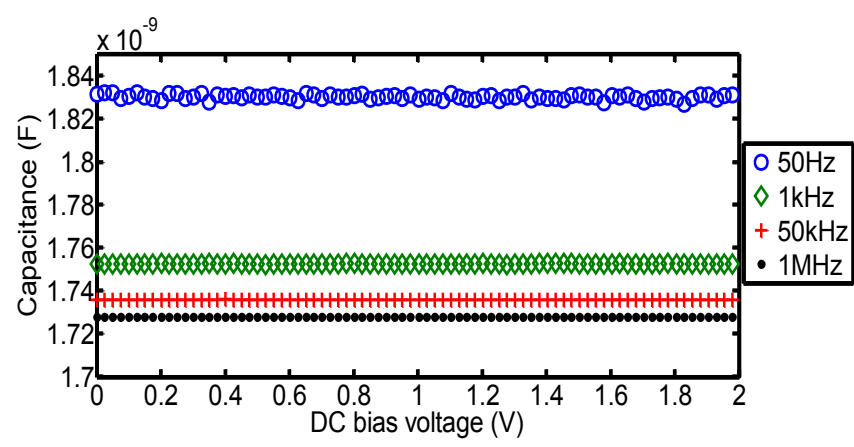

(a)

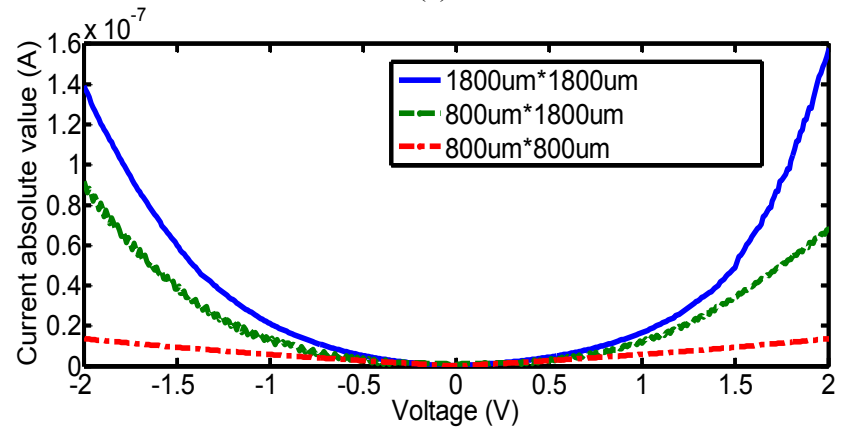

(b)

Figure 11. (a) C-V measurement results for $1800 \mu m \times 1800 \mu m$ size capacitors at various frequencies, (b) $|I|-V$ measurement results

\section{REFERENCES}

[1] The International Technology Roadmap for Semiconductors 2011 edition, Semiconductor Industry Association, 2011.

[2] F. Roozeboom, et al., "Ultrahigh-density trench capacitors in silicon and their application to integrated DC-DC conversion," Proc. 23th Eurosensors Conf., Lausanne, Sept., 2009, pp. 426-430.

[3] J. H. Klootwijk, et al., "Ultrahigh capacitance density for multiple ALD-grown MIM capacitor stacks in 3-D silicon," IEEE Electron Device Letters, vol. 29, no.7, pp. 740 - 742, Jul. 2008.

[4] T. Kaga, et al., "Crown-shaped stacked-capacitor cell for $1.5-\mathrm{V}$ operation 64-Mb DRAMs," IEEE Transactions on Electron Devices, vol. 38, no. 2, pp. 255 - 261, Feb. 1991.

[5] C. T. Black, et al., "Integration of self-assembled diblock copolymers for semiconductor capacitor fabrication," Applied Physics Letters, vol. 79, no. 3, pp. 409-411, Jul. 2001.

[6] T. Tsutsumi, S. Sugitani, K. Nishimura, and M. Ida, "New stacked metal-insulator-metal capacitor with high capacitance density for future InP-based ICs," Japanese Journal of Applied Physics, vol. 50, no. 4, pp. 04DF09-04DF09-4, 2011.

[7] B. Schwartz and H. Robbins, "Chemical etching of silicon II. The system HF, $\mathrm{HNO}_{3}, \mathrm{H}_{2} \mathrm{O}$, and $\mathrm{HC}_{2} \mathrm{H}_{3} \mathrm{O}_{2}$," Journal of The Electrochemical Society, vol. 107, no. 2, pp. 108-111, Feb. 1960.

[8] A. Bajolet, et al., "Low-Frequency series-resistance analytical modeling of three-dimensional metal-insulator-metal capacitors," IEEE Transactions on Electron Devices, vol. 54, no. 4, pp. 742-751, Apr. 2007.

\section{CONTACT}

*Victor Farm-Guoo Tseng, tel: +1-352-328-2344; souldragon227@,ufl.edu 